\title{
Safety training
}

As a teacher and trainer, I am responsible in part for the occupational health knowledge of animal users on my campus. I find the topic of safety to be one of the most difficult to convey to people. It seems that all I'm doing is reciting fact after fact: viruses can make people sick. Personal protective equipment (PPE) appropriate for the task should be worn. HEPA filters don't protect against gases. And so on...

It is challenging to get safety information across and to feel assured that the trainees will be better protected when working in potentially hazardous situations with animals as a result.

We know from Professor Malcolm Knowles that adult learners are less oriented towards the content and more oriented towards the learners-themselves. But safety training is all about the content: the facts and the safe practices. In other words, details matter, and that makes it hard to learn about safety.

I have learned two maxims that describe the learning paradigm and outcome: affect, behavior and cognition (ABC) and knowledge, skill and attitude (KSA). Each has a component that refers to the material to be learned: cognition and knowledge. And each has a component that addresses how a person will act in a situation, if he has the necessary knowledge: behavior and attitude. The third components, affect and skill, don't match so well at first. But affect implies experience or feeling, so it does suggest a level of skill.

I suggest that trainers keep in mind one of these acronyms when teaching information-dense subjects like safety. I can make no apologies when I describe it as rather dry and boring. So, how can we enhance this learning experience? What motivates the adult learner? Let me share one of my strategies.

With my vet tech students, I first give a presentation filled with definitions, pictures, links, etc. Their homework assignment is to visit various websites and find safety equipment that would be needed to protect personnel in a hypothetical lab animal facility of their choice. Both emphasize application of the material and relevance to their interests. Then at the next class meeting, I give a quiz to evaluate their learning as follows:

You are the manager of an animal facility. The institution official (IO) has asked you to develop a training document on safety and sanitation for personnel who use your facility. You decide to write up an outline for her approval first, consisting of several distinct topics and the intended learning (KSA) outcomes. Select 12 topics based upon the contents of last week's safety presentation. Be comprehensive.

To grade the quiz, the students pair up and exchange papers. Each assumes the role of the IO to evaluate the quality of the other's safety 'document' and provide critical feedback. Observing the resulting discussions and knowledge gain will warm any trainer's heart. It's no longer so dry and boring.

I decided to ask two colleagues for their engaging tricks in teaching safety.

Sean Kaufman is a safety training expert with Behavioral-Based Improvement Solutions in Georgia and shared the following tip: "Good trainers are not measured by the number of people they train or the evaluations they receive. Instead, they are measured by observable differences in the practices of the workforce that lead to increases in personal and organizational safety levels. These practices are behaviors that serve as the critical bridge between safety plans and desired outcomes within organizations. Trainers are always training because the word 'trained' does not exist; human error issues such as perceived mastery and complacency remain a constant threat to safety. Trainers promote a culture of safety because they understand how the beliefs of leadership and behaviors of the workforce can influence overall safety practices and compliance. Trainers believe their role is to increase the capacity of those they serve: they 'do' safety with people and by people, not for people or to people, making sure they can do it for themselves. Lastly, if you call yourself a trainer, train with passion. If you are bored providing the content, imagine what someone listening to you is experiencing. Trainers make anything and everything worth it, but only if they love what they do."

Belinda Rivera is a Laboratory Biosafety Training Consultant at University of Texas Medical Branch in Galveston with the National Biocontainment Training Center and is responsible for providing in vitro training for both internal and external trainees working in Animal Biosafety Level 2, 3 and 4 facilities. She writes: "We use a multifaceted approach to our safety training to ensure that our trainees understand the information provided, have the skills needed during an emergency and can perform their work proficiently while maintaining high standards of safety. Our training includes a manual and theoretical lecture that provides trainees with important written information. After completion of the theoretical portion, trainees receive one-on-one, hands-on training to become proficient with the specific species and procedure listed on their IACUC-approved protocol while wearing all appropriate PPE to ensure proper use and fit. Finally, a practical and written assessment is completed by each trainee to confirm proficiency with technical procedures and knowledge of safety protocols. This style of training is very time consuming but has been successful for the highly diverse and multi-cultural staff at our institution. Safety training is not the most exciting material to present, but as a trainer, it is my obligation to make it as informative and interactive as possible to provide trainees with the information and skills needed to keep them safe while performing their important work in high containment facilities."

Let me close with the following: as the safety representative on my IACUC puts in his e-mail signature, "be safe." 